\title{
Effect of $D X$ Centers in the Vertical Transport Properties of Semiconductor Superlattices
}

\author{
F. Aristone ${ }^{\dagger}$, B. Goutiers ${ }^{\ddagger}$, J. L. Gauffier ${ }^{\ddagger}$, and L. Dmowski 9 \\ ${ }^{\dagger}$ DFI-CCET/UFMS, C.P. 549, 79070-900 Campo Grande - MS Brazil \\ ${ }^{\ddagger}$ INSA - Complexe Scientifique de Rangueil, 31077 Toulouse, France \\ \High Pressure Research Center, 01-142 Warsaw, Poland
}

Received 27 September, 1999

\begin{abstract}
$D X$ centers have been detected in vertical transport experiments of $G a A s$-AlAs superlattices. We studied miniband conduction properties of such semiconductor structures in presence of high hydrostatic pressures and controlled temperature. Hystheresis effect in the current-voltage characteristics was observed. We show that miniband transport properties are dependent on the path of the pressure cycle imposed to the sample. It is clear from our results that $D X$ centers are present in the active superlattice region. We propose that the energy associated with $D X$ states in superlattice results from a "hybridization" of $D X$ centers of both GaAs and AlAs bulk materials.
\end{abstract}

Semiconductor superlattices are very attractive structures for use in the design of electronic devices, particularly in high frequency oscillators used in telecommunication systems [1]. Bulk-like negative differential conductance has been demonstrated in vertical transport of superlattices. Electrons moving along growth axis direction are subject to high miniband nonparabolicity and consequently undergo negative effective mass at sufficiently high bias voltage [2].

Band structure of superlattices is more complex than band structure of bulk materials due to the additional periodicity introduced when growing different semiconductors alternately. It is known that $A l_{x} G a_{1-x} A s$ materials present two minima along the [001] direction, the $\Gamma$ and $X$ point usually called $\Gamma$ and $X$ valleys [3]. GaAs has the lowest minimum at the $\Gamma$ valley, which denotes a direct band-gap material. AlAs is an indirect band-gap material because its lowest minimum is located at $X$. Valley mixing on the $G a A s-A l A s$ superlattice interfaces was studied by Ando [4].

Conduction (mini)band structure of superlattices and bulk materials present analogies, since the periodic profiles of $\Gamma$ and $X$ valleys produce the so-called "direct" and "indirect" minibands, respectively [5]. A superlattice is said to be "direct" when the higher energy value for $\Gamma$ miniband is lower than the lowest energy for any $X$ miniband. Relative position of $\Gamma$ and $X$ minibands is a function of well and barrier parame- ters. Symmetric "indirect" GaAs - AlAs superlattices are obtained when well and barrier thickness are less than 13 mono-layers [6]. There are two different $X$ minibands associated with longitudinal and transverse (two-fold degenerate) effective masses.

Shallow and deep levels are also present in the band structure of $A l_{x} G a_{1-x} A s$ materials when the whole structure is doped, e.g., with silicon. Such states arise from the same donor center and are separated by an energy barrier. Shallow levels are explained in terms of effective mass state and deep levels are associated with relaxed donors. Deep donor levels are called $D X$ states and have been observed in III-V semiconductors. In this paper we demonstrate the presence of $D X$ centers in superlattice structures using a vertical transport experiment.

Hydrostatic pressure is frequently used as a very functional tool to explore band structure in semiconductors. Pressure effects in superlattices are more complicated to analyze than they are in bulk materials. Leburton and Kahen studied the pressure coefficient difference of band energy for both bulk materials and superlattices [7]. Warburton et al. investigated the pressure coefficient of effective mass for $G a A s-A l_{x} G a_{1-x} A s$ heterojunctions and found the same value as found in bulk materials, with no dependence on the electron concentration $n$ [8]. Austing et al. studied $\Gamma-X$ tunneling resonance in $G a A s / A l A s$ double barrier under high pressure [9]. 
$\Gamma-X$ electronic intervalley transfer in GaAs $A l_{x} G a_{1-x} A s$ superlattices has been discussed by various authors, either in vertical or parallel transport configuration. Dutisseuil et al. analyzed intra-miniband heating and intervalley scattering applying hydrostaticpressure on superlattices [10]. Nunnenkamp et al. performed time-resolved optical measurements to study transition of type-I to type-II GaAs - AlAs superlattice under pressure [11]. $\Gamma-X$ mixing in $G a A s-A l A s$ has also been studied with the help of a magnetic field by Pulsford et al. [12]. Sellito et al. recently presented a study of $D X$ states in planar doped GaAs - AlAs superlattices [13]. Measuring Hall and photo-Hall effect of uniform and delta-doped superlattices, they showed two main effects of such electronic structure behavior under hydrostatic pressure: 1) miniband crosses over the deep level when high enough pressure is applied; 2) shallow levels are linked to the miniband.

Effects of hydrostatic pressure on vertical transport properties for "direct" and "indirect" GaAs-AlAs superlattices have also been studied. Application of high pressure allows explicit separation of $G a A s-A l A s$ superlattice electronic transport. Transition from "direct" to "indirect" superlattice structures has been demonstrated when sufficiently high hydrostatic pressure is applied to the sample [14]. In this paper we present the first evidence to our knowledge of $D X$ center effect on perpendicular transport of superlattices.

Our samples were grown by molecular beam epitaxy on a $n^{+}$-doped [001] oriented GaAs substrate. The period of sample 1 was equal to $74 \AA$; of which $54 \AA$ was the well and $20 \AA$ the barrier. First $\Gamma$ miniband conduction for this structure was $9 \mathrm{meV}$ wide and separated by $102 \mathrm{meV}$ from the bottom of GaAs conduction band. It is a direct structure (type-I) for which the first $X$ miniband was $62 \mathrm{meV}$ above $\Gamma$ miniband. The period of sample 2 was equal to $34 \AA$; of which $20 \AA$ was the well and $14 \AA$ the barrier. An indirect structure (typeII), its first $X$ miniband was $6 \mathrm{meV}$ wide separated by $80 \mathrm{meV}$ from the bottom of GaAs conduction band. A grading average of the composition was carried out between superlattice active region and doped layers in order to avoid abrupt heterojunctions in the passage of the three-dimensional electron gas from contacts to the miniband.

Conventional lithographic processes were employed to obtain mesa-area typically in the range from $100 \mu \mathrm{m}^{2}$ to $2800 \mu \mathrm{m}^{2}$. AuGeNi was deposited and diffused on both top and bottom $n^{+}$-doped layers, thus giving perfect Ohmic contacts for our samples. The active regions were lightly doped, with a $S i$ concentration of $n^{-} \approx$ $2 \times 10^{16} \mathrm{~cm}^{-3}$ for sample 1 and $n^{-} \approx 1 \times 10^{16} \mathrm{~cm}^{-3}$ for sample 2 , respectively.
Our results were obtained using standard transport technique. All measurements were performed in total absence of light. Hydrostatic pressure was applied using either a liquid clamp cell or a Helium gas compressor. Pressure inside the cell was measured with a calibrated In $\mathrm{Sb}$ resistance.

Typical current-voltage $I(V)$ characteristics were obtained as a function of applied pressure at room temperature and at $T=77 \mathrm{~K}$, for our two samples. These results can in a first approximation be explained as the intervalley electronic transfer, since hydrostatic pressure produces a relative movement of minibands, as discussed by Gassot et al. [15]

However, rigorous observation of $I(V)$ characteristics, in particular for "indirect" sample, indicates that more precise arguments are necessary to explain our data. We plot in Fig. $1 I(V)$ characteristics for different temperatures, obtained at constant pressure $P=12 \mathrm{kbar}$. The current intensity decreases monotonically as the temperature decreases. Such a decrease in current cannot be explained as intervalley transfer, because this sample is already "indirect" at atmospheric pressure. Therefore it is evident that no electrons are present in the $\Gamma$ miniband at high pressure, even at room temperature.

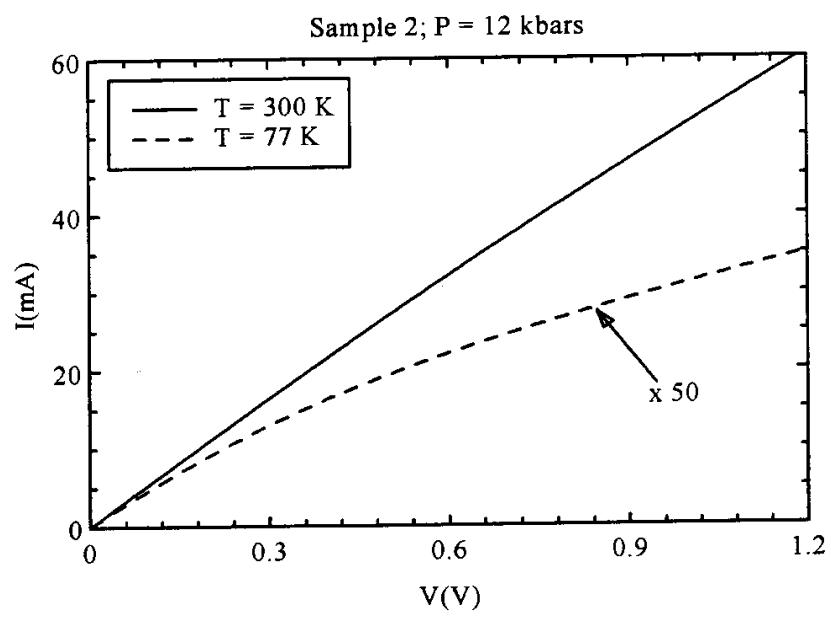

Figure 1. $I(V)$ characteristics at constant pressure $P=$ 12 kbars for "indirect" sample 2. Two different temperatures are shown. Dashed curve has been multiplied by factor 50, to appear in the same scale. Decrease in current intensity is explained as electron trapping by $D X$ centers.

To explain this effect we need to consider the presence of recombination centers in the superlattice structure. Current intensity decreases because electrons remain confined to trapping states when temperature goes down. We can also infer that the pressure coefficient of this recombination center is very close to the pressure coefficient of $X$ miniband. In order to study quantitatively the presence of a recombination level in 
our structure, we performed a "cycle of pressure" experiment, which is divided in two parts as described below:

\begin{tabular}{|l|l|}
\hline First Part & Second Part \\
\hline $\begin{array}{l}\text { i) } I_{1}(V) \text { characteristic is measured at room tem- } \\
\text { perature } T_{r}=300 \mathrm{~K} ;\end{array}$ & $\begin{array}{l}\text { I) } I_{4}(V) \text { characteristic is measured at room tem- } \\
\text { perature } T_{r}=300 \mathrm{~K} ;\end{array}$ \\
\hline $\begin{array}{l}\text { ii) sample is cooled to } T_{n}=77 \mathrm{~K} ; I_{2}(V) \text { character- } \\
\text { istic is measured; }\end{array}$ & $\begin{array}{l}\text { II) Pressure is applied up to } P_{m}=12 \mathrm{kbars}, \text { while } \\
\text { temperature } T_{r}=300 \mathrm{~K} \text { is kept constant; } I_{5}(V) \\
\text { characteristic is measured; }\end{array}$ \\
\hline $\begin{array}{l}\text { iii) sample is heated to } T_{r}=300 \mathrm{~K} ; I_{3}(V) \text { charac- } \\
\text { teristic is measured. }\end{array}$ & $\begin{array}{l}\text { III) temperature is cooled to } T_{n}=77 \mathrm{~K} \text { and pres- } \\
\text { sure } P_{m}=12 \mathrm{kbars} \text { is maintened constant; } I_{6}(V) \\
\text { characteristic is measured; }\end{array}$ \\
\hline & $\begin{array}{l}\text { IV) pressure is completely relaxed with tempera- } \\
\text { ture } T_{n}=77 \mathrm{~K} \text { constant; } I_{7}(V) \text { characteristic is } \\
\text { measured; }\end{array}$ \\
\hline & $\begin{array}{l}\text { V) sample is heated to } T_{r}=300 \mathrm{~K} ; I_{8}(V) \text { charac- } \\
\text { teristic is measured. }\end{array}$ \\
\hline
\end{tabular}

Such a cycle is schematically represented on $P-T$ diagram of Fig. 2. $I_{1}(V), I_{3}(V), I_{4}(V)$ and $I_{8}(V)$ characteristics were measured (on position $R$ of Fig. 2) to determine whether irreversible deformation occurred in the sample or not. No variation has been observed among all these characteristics, insuring that intrinsic electronic properties of superlattice were preserved during the process.

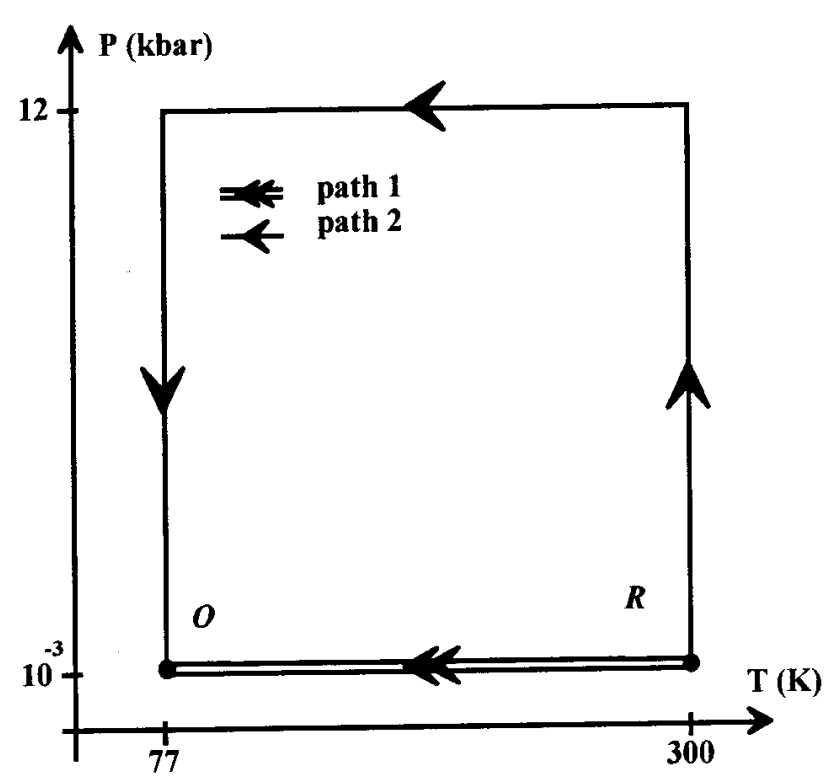

Figure 2. Schematic representation of the "pressure cycle" experiment. Vertical transport properties of GaAs-AlAs superlattices were measured during the cycle. Results obtained at position $R$ certify that hydrostatic pressure did not damage the sample. We compared $I(V)$ characteristics at point $O$ for two different paths and hystheresis behavior was observed.

Transport properties are not identical, however, at point $O$ due to presence of $D X$ centers in superlattice structure. We compare $I_{2}(V)$ and $I_{7}(V)$ characteristics that were obtained both at atmospheric pressure and at $T=77 \mathrm{~K}$, but measured at the end of different paths on the "cycle of pressure". Hystheresis behavior observed for sample 1 is exhibited in Fig. 3. This effect is also direct evidence for the presence of $D X$ centers in the superlattice structure.

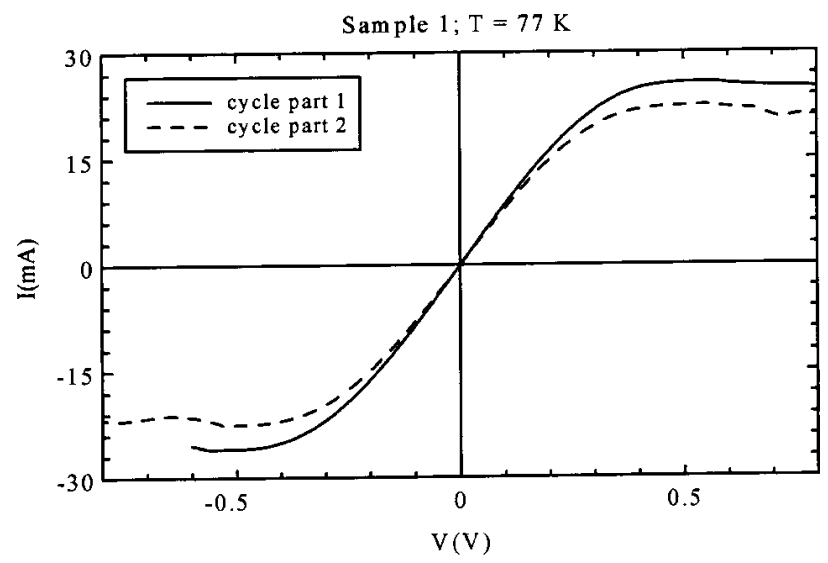

Figure 3. $I(V)$ characteristics obtained for sample 1 at point $O$ of the "pressure cycle" experiment, which is represented in Fig. 2 and described in the text. Hystheresis behavior is due to trapping of electrons due to $D X$ centers present in the active region of superlattice.

Hydrostatic pressure was applied at room temperature and due to high thermal energy electrons are distributed among $\Gamma$ and $X$ minibands and deep centers. When temperature decreases at constant pressure, less electrons have energy to access the high miniband. At $T=77 \mathrm{~K}$ no more electrons are present in $\Gamma$ miniband. Relaxing of pressure (now at constant temperature) replaces $\Gamma$ miniband as the lower energy value. Thus no 
more electrons have access either to $X$ miniband or to $D X$ states. Some electrons, however, remained trapped in $D X$ states due to low thermal energy. Hystheresis effect is observed between these two particular measurements of $I(V)$ characteristics. Presence of $D X$ centers in the superlattice structure is therefore made evident.

We estimate the energy position, entropy factor and pressure coefficient of this energy trapping level, based on the Theis [16] and Adachi [17] description of $D X$ center. We concluded for sample 2 that: 1) a $D X$-type recombination center is located approximately $100 \mathrm{meV}$ below the miniband $X ; 2)$ the entropy factor is $S \approx-0.2 \mathrm{meV} / \mathrm{K} ; 3)$ its pressure coefficient is close to pressure coefficient of miniband $X$, roughly equal to $10 \mathrm{meV} / \mathrm{kbar}$. We also calculated doping concentration in the active structure and we found $N_{d} \sim 0.8 \times 10^{16} \mathrm{~cm}^{-3}$, which is in substantial agreement with growth parameters. Results for sample 1 lead to equal entropy factor and pressure coefficient. We estimate in this case that a $D X$ level is located at $120 \mathrm{meV}$ below the $X$ miniband.

We have characterized, therefore, a recombination state in our structures for which the pressure coefficient, entropy factor and energy position are identical to bulk $D X^{0}$ "relaxation level" values. The "cycle of pressure" expresses this same behavior, i.e., hystheresis observed identifies a lattice relaxation level.

In conclusion, we demonstrated the presence of deep levels in $G a A s / A l A s$ superlattices using vertical transport experiments in presence of high hydrostatic pressure. Two principal effects were observed: decrease of current intensity as a function of temperature for indirect samples and hystheresis behavior signifying the presence of a $D X$ level. After determination of energy position, pressure coefficient and entropy factor we associated such a state with a $D X^{0}$ level. We proposed the presence of recombination level in superlattices due to "hybridization" of $D X$ centers of both $\mathrm{GaAs}$ and AlAs materials.

\section{Acknowledgments}

We express special gratitude to J. F. Palmier and J.-C. Portal for a critical reading of this paper. We acknowledge P. Sikar for comments, J. C. Esnault and S. Vuye for technical support. One of us (F. A.) is particularly grateful to $\mathrm{CNP}_{\mathrm{q}}$-Brasil for financial support. We carefully acknowledge the samples provided by FranceTelecom/CNET.

\section{References}

[1] M. Hadjazi, J. F. Palmier, A. Sibille, H. Wang, E. Paris and F. Mollot. Electronics Letters 29, 8:684 (1993).

[2] A. Sibille, J. F. Palmier, H. Wang and F. Mollot. Phys. Rev. Lett. 64, 52 (1990).

[3] M. Guzzi and J. L. Staehli. Solid State Phenomena 10, 25 (1989).

[4] T. Ando. Phys. Rev. B 47, 9621 (1993).

[5] O. E. Raichev. Sov. Phys. Semicond. 25, 740 (1991).

[6] K. Reimann. Jpn. J. of Appl. Phys. 32, 64 (1993).

[7] J. P. Leburton and K. Kahen. Superlatt. Microstruct. 1, 49 (1985).

[8] R. J. Warburton, M. Watts, R. J. Nicholas, J. J. Harris and C. T. Foxon. Semicond. Sci. Technol. 7, 787 (1992).

[9] D. G. Austing, P. C. Klipstein, A. W. Higgs, H. J. Hutchinson, G. W. Smith, J. S. Roberts and G. Hill. Phys. Rev. B 47, 1419 (1993).

[10] E. Dutisseuil, A. Sibille, J. F. Palmier, F. Aristone, F. Mollot and V. Thierry-Mieg. Phys. Rev. B 49, 5093 (1994).

[11] J. Nunnenkamp, K. Reimann, J. Kuhl and K. Ploog. Surface Science 263, 553 (1992).

[12] N. J. Pulsford, R. J. Nicholas, P. Dawson, K. J. Moore, G. Duggan, and C. T. B. Foxon. Phys. Rev. Lett. 63, 2284 (1989).

[13] P. Sellito, J. Sicart, J. L. Robert and R. Planel. Phys. Rev. B 51, 16778 (1995). More recently: Miwa and Schmidt, Applied Physics Letters 74, 1999 (1999).

[14] F. Aristone, A. Sibille, J. F. Palmier, B. Goutiers, J. C. Portal and F. Mollot. Jpn. J. Appl. Phys. 32, 144 (1993).

[15] P. Gassot, D. K. Maude, M. Eremets, F. Aristone, B. Goutiers, J. L. Gauffier, J. F. Palmier, J. C. Portal, J. C. Harmand and F. Mollot. Solid State Electronics 40, 185 (1996).

[16] Theis, Mooney and Parker. Phys. Rev. Lett. 60, 361 (1988). E. Calleja, A. Gomez and E. Muñoz. Appl. Phys. Lett. 52, 383 (1988).

[17] Sadao Adachi. J. Appl. Phys. 58, R1 (1985). 